Revue internationale P.M.E.

Économie et gestion de la petite et moyenne entreprise

Revue

internationale

PME

\title{
La représentation identitaire de la TPE artisanale
}

\section{Christian Picard}

Volume 19, numéro 3-4, 2006

URI : https://id.erudit.org/iderudit/1008500ar

DOI : https://doi.org/10.7202/1008500ar

Aller au sommaire du numéro

Éditeur(s)

Presses de l'Université du Québec

ISSN

0776-5436 (imprimé)

1918-9699 (numérique)

Découvrir la revue

Citer cet article

Picard, C. (2006). La représentation identitaire de la TPE artisanale. Revue internationale P.M.E., 19(3-4), 13-49. https://doi.org/10.7202/1008500ar

\section{Résumé de l'article}

À partir du paradoxe selon lequel l'artisanat, « première entreprise de France ", constitue un objet d'observation important tout en étant relativement mal connu et peu exploré, cet article propose de décrire la nature de chacune des entreprises qui composent cet ensemble institutionnel. Le but affiché est alors de contribuer à une meilleure intelligibilité de la TPE artisanale en tant que forme d'organisation spécifique, notamment en termes de fonctionnement et de pilotage.

En prenant appui sur la transposition au cas de la TPE artisanale, de l'approche identitaire, initialement destinée aux grandes organisations, une représentation conceptuelle du système identitaire de l'entreprise artisanale est proposée sous la forme d'un système identitaire particulier. Cette représentation est ensuite validée par une étude empirique réalisée auprès d'un échantillon de 346 artisans. Les résultats conduisent notamment à mettre en relief deux configurations identitaires concurrentes : celle de l'artisan et celle de l'entrepreneur. 


\title{
La représentation identitaire de la TPE artisanale
}

\author{
Christian PICARD
}

Institut de recherche en gestion et en économie (IREGE)

Université de Savoie

\section{MOTS CLÉS}

\section{Artisanat - Entreprise artisanale - Identité - Métier Système identitaire - Configurations empiriques}

\begin{abstract}
L'AUTEUR
ChRISTIAN PICARD est maître de conférences en sciences de gestion à l'Université de Savoie; il est membre de l'Institut de recherche en gestion et en économie (IREGE) et occupe les fonctions de chef du Département Gestion administrative et commerciale à l'IUT de Chambéry. Son champ de recherche concerne les très petites entreprises (TPE) et les entreprises artisanales. Ses travaux portent notamment sur les dynamiques d'évolution de l'entreprise artisanale, thème de sa thèse de doctorat, sur les problèmes liés à la transmission et la reprise d'entreprises ainsi que sur les questions du changement identitaire des métiers artisanaux. Adresse : IREGE, Université de Savoie, B.P. 240, 74942 Annecy-le-Vieux, France. Courriel: <christian.picard@univ-savoie.fr>.
\end{abstract}

\section{RÉSUMÉ}

À partir du paradoxe selon lequel l'artisanat, «première entreprise de France», constitue un objet d'observation important tout en étant relativement mal connu et peu exploré, cet article propose de décrire la nature de chacune des entreprises qui composent cet ensemble institutionnel. Le but affiché est alors de contribuer à une meilleure intelligibilité de la TPE artisanale en tant que forme d'organisation spécifique, notamment en termes de fonctionnement et de pilotage.

En prenant appui sur la transposition au cas de la TPE artisanale, de l'approche identitaire, initialement destinée aux grandes organisations, une représentation conceptuelle du système identitaire de l'entreprise artisanale est proposée sous la forme d'un système identitaire particulier. Cette représentation est ensuite validée par une étude empirique réalisée auprès d'un échantillon de 346 artisans. Les résultats conduisent notamment à mettre en relief deux configurations identitaires concurrentes: celle de l'artisan et celle de l'entrepreneur. 


\section{ABSTRACT}

From the paradox according to which the crafts, "first company of France", constitute an important object of observation relatively badly known and little investigated, this article suggests becoming attached to the nature of each of the companies which compose this institutional group. The purpose is then to contribute to a better comprehensibility of the craft TPE, as shape of specific organization, notably in terms of functioning and piloting.

By taking support, for the case of the craft TPE, on the transposition of the identity approach, initially dedicated in big organization, an abstract representation of the identity's system of the craft company is proposed under the shape of a particular identity system.

This representation is then validated by an empirical study realized with a sample of 346 craftsmen. The results notably lead to accentuate two rival identity's configurations : that of the craftsman and that of the entrepreneur.

\section{RESUMEN}

A partir de la paradoja según la cual el artesanado, «Primera empresa de Francia » constituye un objeto de observación importante aunque relativamente mal conocido y poco explorado, este artículo propone tratar de la naturaleza de cada una de las empresas que componen este conjunto institucional. El objetivo es pues contribuir a una mejor inteligibilidad de las muy pequeñas empresas (TPE) artesanales, como forma de organización específica, principalmente en términos de funcionamiento y pilotaje.

Tomando como base la transposición al caso de la muy pequeña empresa artesanal, del enfoque de identidad, inicialmente utilizado para las grandes organizaciones, se propone una representación conceptual del sistema de identidad de la empresa artesana en base de un sistema de identidad particular. Esta representación es confirmada después por un estudio empírico realizado sobre una muestra de 346 artesanos. Los resultados conducen a poner de relieve principalmente dos configuraciones de identidad competidoras: la del artesano y la del empresario.

\section{ZUSAMMENFASSUNG}

Obwohl das Handwerksgewerbe, 'wichtigste Unternehmensform Frankreichs', ein interessantes Beobachtungsobjekt für die Wissenschaft darstellt, wurde es bis anhin wenig erforscht und ist aus diesem Grunde eher unbekannt. Der vorliegende Artikel versucht die Eigenheiten der einzelnen Unternehmen zu verstehen, um die institutionelle Gesamtheit des Handwerkgewerbes zu erfassen. Ziel ist mithin, zu einem besseren Verständnis über die Funktionsweise und die Führung der kleinen Handwerksbetriebe beizutragen.

Aufbauend auf dem ursprünglich für grosse Organisationen entwickeln Identitätsansatz (Identität der Branche), kann ein spezifisch für die kleinen Handwerksbetriebe erarbeitetes Identitätssystem präsentiert werden. Das entwickelte Konzept wurde anschliessend an einer Stichprobe von 346 Handwerksbetrieben empirisch

Revue internationale P.M.E., vol. 19, $\mathrm{n}^{\text {os }}$ 3-4, 2006

(C) 2006 - Presses de l'Université du Québec

Édifice Le Delta I, 2875, boul. Laurier, bureau 450, Québec, Québec G1V 2M2 • Tél.: (418) 657-4399 - www.puq.ca

Tiré de: Revue internationale P.M.E., vol. 19, $\mathrm{n}^{\text {os }} 3-4$, sous la direction de Louis Raymond • PME1903N Tous droits de reproduction, de traduction et d'adaptation réservés 
geprüft. Die Ergebnisse zeigen auf, dass zwei sich konkurriende Identitätskonfigurationen gebildet werden können, nämlich jene des Handwerkers und jene des Unternehmers.

\section{Introduction}

L'artisanat en France constitue un objet d'observation important puisqu'il compte 850000 entreprises et emploie $10 \%$ de la population active française (DEcas, 2004). C'est également un domaine d'investigation bien défini, sur le plan juridique et institutionnel, par des critères d'activité et de taille des entreprises qui lui confèrent une apparente homogénéité interne et différencient les entreprises qui le composent des autres. Paradoxalement, il reste encore mal connu et mal délimité. Il est vrai que l'hétérogénéité qui caractérise ce microcosme fait obstacle à la généralisation des recherches dans ce domaine ainsi qu'à l'élaboration d'une représentation théorique robuste de l'entreprise artisanale. Malgré sa réémergence en tant qu'objet d'observation et de recherche (Auvolat, 1990), dans la foulée du renouveau des travaux sur les PME, les réflexions qui lui sont consacrées butent toujours sur un problème de définition de l'objet étudié. Cela se traduit alors par une multiplicité des champs de vision existants. Bien qu'une «théorie de la PME» commence à se dessiner (Julien, 1994), la TPE et l'entreprise artisanale n'ont pas encore atteint ce degré d'autonomie et sont encore souvent considérées comme des «modèles réduits» des PME (Marchesnay, 2003). Ainsi, les travaux prenant en compte la spécificité de ces organisations et destinés à les aider dans la résolution de leurs difficultés de gestion sont rares. Plus précisément, c'est la prise en compte de l'entreprise artisanale en tant qu'objet d'observation qui fait défaut. Dans ce contexte, cet article propose un nouvel éclairage en matière de connaissance de la spécificité de l'entreprise artisanale. Il plaide pour une spécificité identitaire de la firme artisanale en adoptant le présupposé suivant: ce qu'elle est (ontologie) conditionne ce qu'elle fait, comment elle se comporte sur le plan organisationnel mais aussi dans son environnement. Pour cela, la démonstration suit deux étapes: tout d'abord, l'élaboration d'un cadre méthodologique cherchant à concilier les connaissances disponibles sur l'artisanat et les TPE-PME avec le discours théorique sur l'identité de l'entreprise; ensuite, la validation de la représentation identitaire proposée en s'appuyant sur les résultats d'une importante étude quantitative auprès d'artisans. 


\section{La spécificité du système identitaire artisanal: références conceptuelles et cadre d'analyse}

L'élaboration de notre cadre d'analyse se fera en trois étapes. Premièrement, montrer succinctement que la littérature consacrée à l'artisanat n'apporte qu'un éclairage partiel sur la spécificité de ce type d'entreprise et présenter la métaphore identitaire de l'organisation comme un outil de description approprié pour fédérer les différentes approches proposées dans cette littérature sur l'artisanat. Deuxièmement, proposer notre représentation identitaire de la firme artisanale sous la forme d'un «idéal-type». Enfin, identifier les différentes configurations possibles de cet idéal-type et faire émerger les hypothèses que l'étude quantitative cherchera à valider

\subsection{L'incomplétude des travaux consacrés à l'artisanat et l'intérêt de la métaphore identitaire}

En parcourant le répertoire des publications sur l'artisanat ${ }^{1}$, on ne peut que constater la diversité qui caractérise la littérature qu'il recense. Au premier abord, l'hétérogénéité des études et recherches contemporaines est presque aussi grande que celle qui caractérise l'objet observé. Il est toutefois possible d'identifier certaines lectures dominantes du «phénomène artisanal », correspondant à différentes disciplines des sciences humaines telles que le droit, l'économie, la sociologie ou la gestion et présentant des approches différentes d'une même réalité. Devant une telle diversité d'analyse, la démarche adoptée a consisté, dans un premier temps, à recenser et à identifier les principaux travaux réalisés dans chacun de ces champs disciplinaires. Les différentes conceptions relevées ont ensuite été étudiées selon le fil directeur suivant: quelles sont leurs logiques d'analyse et leurs caractéristiques distinctives? Pour finalement répondre à la question: quelles sont leurs contributions respectives à la connaissance de la spécificité de l'entreprise artisanale? Le tableau ci-dessous présente de manière synthétique ces résultats.

1. Si de nombreux travaux réalisés avant la fin des années 1960 restent, faute de publication, dans une grande confidentialité et sont, pour la plupart, inaccessibles, les écrits plus récents sont plus facilement repérables, notamment grâce au «Répertoire bibliographique des documents publiés sur l'artisanat depuis 1970 » et disponible d'abord au sein du Centre d'études et de perfectionnement sur l'artisanat puis au sein de l'Institut supérieur des métiers. Il faut ajouter celui du service des études de la Direction de l'artisanat. 


\section{TABLEAU 1}

\section{Les principaux champs disciplinaires d'analyse de l'artisanat}

\begin{tabular}{|c|c|}
\hline Disciplines et auteurs principaux & $\begin{array}{l}\text { Logique d'analyse } \\
\text { et caractéristiques distinctives }\end{array}$ \\
\hline $\begin{array}{l}\text { Droit } \\
\text { Bénard (1927)-Chaudieu (1982) } \\
\text { Debré (1934)-Mignot (1980) } \\
\text { Décrets et textes officiels parus au } \\
\text { Journal officiel (JO) }\end{array}$ & $\begin{array}{l}\text { Vision collective et réglementaire. L'arti- } \\
\text { sanat est un groupe institutionnel (le secteur } \\
\text { des métiers), juridiquement encadré par } \\
\text { des critères de taille, d'activité et de mode } \\
\text { d'exercice de ces activités. }\end{array}$ \\
\hline $\begin{array}{l}\text { Économie } \\
\text { Auvolat, Lavigne et Mayère (1985) } \\
\text { Arena et al. (1982) - Jaeger (1982) } \\
\text { INSEE et études CEPAM/ISM }\end{array}$ & $\begin{array}{l}\text { Vision collective et fonctionnaliste: l'ar- } \\
\text { tisanat est un «secteur» au service de } \\
\text { l'industrie. Il se distingue de cette dernière } \\
\text { sur le mode de production pratiqué, son posi- } \\
\text { tionnement au sein des filières de production } \\
\text { et son rôle économique. }\end{array}$ \\
\hline $\begin{array}{l}\text { Sociologie } \\
\text { Abric et Mardella (1983) } \\
\text { Froger et Weiller (1986) - Voisin } \\
(1986) \\
\text { Letowski (1987) - Zarca (1982, 1986, } \\
1987,1988)\end{array}$ & $\begin{array}{l}\text { Vision collective et fonctionnaliste. L'arti- } \\
\text { sanat est un groupe social homogène, doté } \\
\text { d'un rôle social d'intermédiation entre } \\
\text { salariat et patronat et porteur d'une identité } \\
\text { particulière, vecteur d'autoreproduction et } \\
\text { d'adaptation. }\end{array}$ \\
\hline $\begin{array}{l}\text { Gestion } \\
\text { Louart (1985)-Fourcade (1993) } \\
\text { Jarniou et Léger (1989)-Marchesnay } \\
(1980,1982,1985,1991,1993,2003 \text {, } \\
\text { 2005) } \\
\text { ERFI et GREPME }\end{array}$ & $\begin{array}{l}\text { Vision plus individualiste et intervention- } \\
\text { niste. L'entreprise artisanale est un système } \\
\text { de gestion spécifique dont la logique d'action } \\
\text { est déterminée par les buts du dirigeant et } \\
\text { dont le mode d'organisation s'appuie sur le } \\
\text { triptyque artisan-compagnon-apprenti. }\end{array}$ \\
\hline
\end{tabular}

La lecture de cet ensemble de contributions conduit à deux conclusions : la première est que la majorité des travaux s'intéresse davantage à l'artisanat ou au «système artisanal» en général qu'aux entreprises qui le composent. Ces différentes approches se heurtent alors à une hétérogénéité qui rend peu représentatif tout discours général sur l'artisanat. Ensuite, l'autre partie des travaux tombe dans l'excès inverse en focalisant son attention sur des métiers ou des activités particulières, perdant alors toute représentativité dans le secteur des métiers.

En résumé, l'objet même d'observation reste à construire. En effet, aucune des approches «disciplinaires» étudiées ne permet de saisir, à elle seule, le système artisanal dans sa globalité et sa complexité et, partant, de fournir un cadre d'analyse adapté à l'étude de l'entreprise artisanale. Chacune 
fournit toutefois des éléments qui éclairent, peu ou prou, la spécificité de l'entreprise artisanale. Toute la difficulté réside alors dans la manière dont on peut les associer, les combiner et les articuler.

Dans cette perspective, afin d'enrichir les problématiques existantes, notamment celle des gestionnaires, et plutôt que de se limiter au constat d'un fonctionnement particulier de ce type d'entreprise, c'est dans sa nature même, dans l'identification des sous-systèmes pertinents pour la décrire et la mise en lumière de leurs caractéristiques respectives, que nous proposons de chercher cette spécificité. Pour ce faire, la mobilisation de la métaphore identitaire (initialement développé pour les grandes entreprises dans le courant des années 1980) nous a paru adaptée à cet objectif. Dans le cadre d'une réflexion sur la définition de l'organisation qui est en définitive un questionnement sur la nature de l'existant et l'acquis du passé, la métaphore identitaire constitue alors une construction et un outil intellectuel privilégié de la perception globale de cette organisation. Il s'agit d'une façon de penser et de se représenter l'organisation qui s'inscrit dans le prolongement de l'analogie entreprise - organisme vivant et dans laquelle chaque entreprise a une identité qui lui est propre et qui contribue à faire de son système de gestion quelque chose d'original et d'unique ${ }^{2}$.

Si l'on admet que l'identité d'une organisation peut être vue comme le lien ou l'interface entre l'intérieur et l'extérieur de cette organisation, elle peut alors être considérée comme un élément assurant la cohérence de ce que l'on appellera, à l'instar de Hellriegel, Slocum et Woodman (1989) ou encore Rojot et Bergmann (1989), le «comportement organisationnel ${ }^{3} »$. Appliquée à l'entreprise artisanale, l'identité permet de «mettre en cohérence» deux caractéristiques majeures du comportement organisationnel de ce type d'entreprise, qui émergent des travaux sur l'artisanat évoqués plus avant:

- L'artisanat est une réalité sociologique particulière. Au regard du système artisanal comme à celui de chacune des entités qui le composent, cette particularité repose, pour l'essentiel, sur des rapports humains d'un type particulier, une conscience partagée d'une position sociale spécifique ou perçue comme telle, et sur la prépondé-

2. Appliqué à l'individu, le terme d'identité correspond au «sentiment d'exister en tant qu'être cohérent et spécifique, assumant son passé, ayant sa place dans la collectivité et reconnu comme tel par les autres» (Laufer et Ramanantsoa, 1982). Transposée aux organisations, cette notion permet de répondre à une série de questions qui sont: Qui est-elle? Quelles sont ses différences? Quelle est sa réalité permanente? Où se situe sa singularité? (Kapferer, 1988; Nizard,1983; Zemor, 1984.)

3. La définition large de cette notion englobe, d'une part, le comportement de l'organisation dans son environnement et, d'autre part, le comportement des individus au sein de l'organisation. 
rance de l'homme (l'artisan), notamment par rapport aux machines ${ }^{4}$. La capacité du concept d'identité à prendre en compte la dimension humaine dans l'organisation en fait alors un outil privilégié pour aborder cette spécificité, notamment parce qu'il autorise la mise en relation des éléments culturels avec les buts et objectifs entrepreneuriaux et avec le «design organisationnel» correspondant.

- L'entreprise artisanale entretient des rapports particuliers avec son environnement. Cette relation, principalement placée sous le signe de la dépendance, est également un élément qui contribue à la spécificité de ce type d'organisation. Elle traduit en fait une caractéristique importante du métier stratégique de l'entreprise artisanale. En autorisant l'introduction de ce «concept relais» dans l'analyse, la métaphore identitaire permet, d'une part, d'effectuer un diagnostic du métier stratégique de l'entreprise artisanale et, d'autre part, d'en proposer une explication: les caractéristiques «externes» du métier stratégique sont alors la résultante de l'interaction des autres éléments contribuant à l'identité de l'entreprise (projets, objectifs et finalités du dirigeant, image interne de l'entreprise et du métier, culture).

À l'aide du modèle de repérage de l'identité d'une entreprise proposé par Larçon et Reitter (1979b), on a tenté de mettre en relation cet univers conceptuel de l'identité avec celui, peut-être plus tangible, de l'artisanat. Le schéma suivant propose ainsi un premier rapprochement des caractéristiques propres à l'entreprise et au milieu artisanal avec chacun de ces sous-systèmes. Il débouche sur un «idéal-type» du système identitaire artisanal ${ }^{5}$.

4. On fait ici référence à l'ensemble des travaux d'essence sociologique qui se sont intéressés à l'artisanat et, plus particulièrement, à la contribution de Zarca dans ce domaine. La caractérisation de l'entreprise artisanale en tant que système de gestion centré sur les objectifs du dirigeant (Julien et Marchesnay, 1988) peut également être rattachée à cette idée.

5. Cette démarche permet un premier repérage de «l'identité artisanale». Le caractère stable sinon immuable, sur longue période, de certaines caractéristiques de l'entreprise artisanale (structure, produits-marchés, chiffre d'affaires, etc.) que révèlent les études empiriques, justifie cette méthode et atténue les inconvénients d'une limitation temporelle et historique de l'analyse. Cette position est d'autant plus défendable que parmi les quatre groupes de facteurs auxquels nous allons nous intéresser, deux forment le fondement de l'identité et peuvent, à ce titre, être considérés comme présents dès la création de l'entreprise. 


\section{Figure 1}

\section{Le système identitaire artisanal}

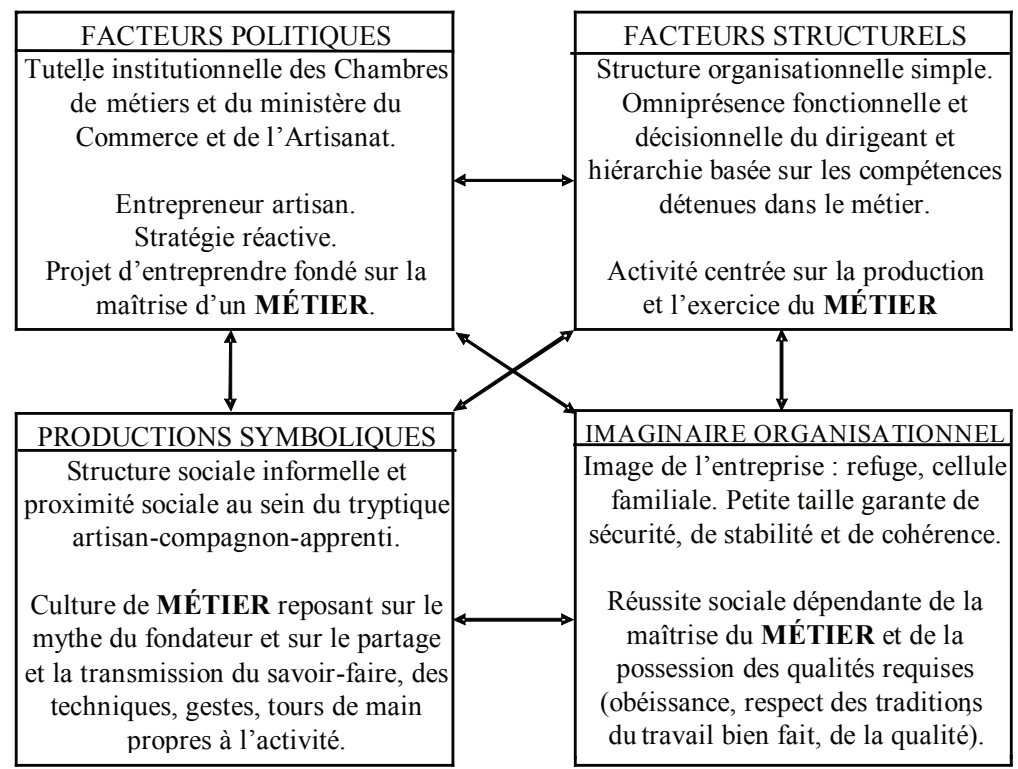

Source: Adapté de Larçon et Reitter (1979b).

Cependant, en suivant cette grille d'analyse, on retrouve alors la confusion ou le chevauchement relevé précédemment: l'analyse du secteur artisanal se substitue à l'analyse des firmes qui composent ce secteur. Si cette démarche autorise une caractérisation identitaire du secteur ou du milieu artisanal, elle ne permet pas de capter l'essence du système identitaire de la firme artisanale. Certes, les caractéristiques identitaires du secteur artisanal «jouent» sur chaque entreprise, mais elles jouent pareillement sur chacune d'elles, de telle sorte qu'elles perdent de leur pertinence pour établir l'identité propre d'une firme artisanale. Afin de surmonter cette difficulté, on propose de distinguer, d'un côté, les caractéristiques identitaires partagées «collectivement» par les entreprises de l'artisanat et constitutives de l'identité du secteur institutionnel des métiers et, de l'autre, les éléments constitutifs du système identitaire de chaque firme artisanale qui définissent le noyau identitaire $^{6}$ de ce type d'organisation.

6. Le «noyau identitaire» de l'entreprise artisanale doit être entendu comme l'ensemble des «caractéristiques identitaires essentielles» qui, d'une part, donnent à chaque entreprise artisanale sa spécificité et, d'autre part, permettent d'obtenir une définition identitaire satisfaisante de chacune de ces entreprises (Mucchielli, 1992). 


\subsection{Le cadre d'analyse du système identitaire de l'entreprise artisanale}

Sans toutefois remettre en cause l'intérêt conceptuel et méthodologique du modèle identitaire, ni son architecture générale, son application à l'entreprise artisanale nous conduit donc à en extraire le noyau identitaire. Le système identitaire de la firme artisanale comporte toujours quatre sous-systèmes ou pôles en interaction comprenant des caractéristiques objectives et subjectives de l'identité et autorisant une définition ontologique de la firme. En revanche, ces pôles diffèrent sensiblement de ceux présents dans la version initiale du modèle. Comme le montre la figure ci-dessous, notre représentation de ce système identitaire particulier retient le profil du dirigeant, son projet d'entreprendre et ses objectifs stratégiques, sa perception du métier et sa perception de l'environnement comme les constituants d'un cadre général d'analyse de l'identité de la firme artisanale. Le sens où chacun doit être entendu est explicité ici, en même temps que les raisons qui justifient leur choix et que les éléments permettant l'opérationnalisation de chaque sous-système.

\section{Figure 2}

\section{Le système identitaire de l'entreprise artisanale}

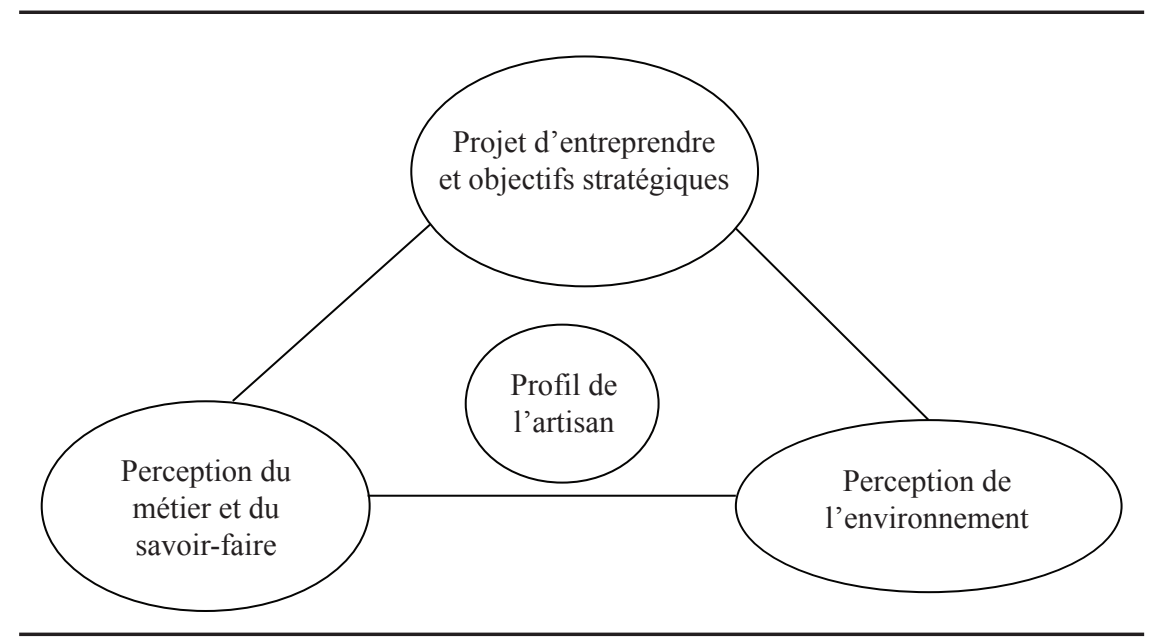

Revue internationale P.M.E., vol. 19, $\mathrm{n}^{\text {os }} 3-4,2006$ 
Nous retenons tout d'abord l'idée que c'est l'artisan qui fait l'entreprise artisanale. En accord avec les études sur l'entrepreneuriat dans les PME et $\mathrm{TPE}^{7}$, le système de gestion de ce type d'organisation est donc centré sur l'artisan. Si l'on cherche à capter la spécificité de ce système de gestion par son identité, l'artisan doit alors être placé au cœur du système identitaire de l'entreprise artisanale. Par conséquent, notre cadre d'analyse du système identitaire de l'entreprise artisanale accordera une place centrale au dirigeant en considérant qu'il joue un double rôle. Tout d'abord, un rôle de relais entre le mésosystème artisanal et l'intérieur de son organisation, notamment en matière de diffusion et de reproduction des valeurs et des comportements dominants de l'artisanat. Ensuite, un rôle de filtre (à travers sa personnalité), puisque c'est de sa propre perception d'une série d'éléments, qui vont d'ailleurs constituer les autres pôles de notre modèle, que va émerger l'identité de son entreprise dans ce qu'elle a de spécial et d'unique. C'est à partir de variables révélant son profil et son identité socioprofessionnelle (âge, sexe, milieu d'origine, situation professionnelle avant l'installation, formation, qualification) mais également à partir de son historique dans l'entreprise (en est-il le fondateur? sous quelle forme? depuis quand?) que l'on cherchera à documenter ce pôle. Il s'agira notamment de distinguer les dirigeants qui ont suivi le «cheminement artisanal» classique (apprenti - compagnon - chef d'entreprise) de ceux plus extérieurs au secteur des métiers ayant un profil de manager ou de gestionnaire.

Dans le modèle de Larçon et Reitter, l'identité de l'entreprise se fonde également sur les objectifs stratégiques de celle-ci et donc sur ceux du ou des dirigeants. Dans les PME, les TPE et a fortiori dans l'artisanat, l'entreprise est, pour son dirigeant, un moyen d'atteindre ses propres buts (Saporta,1989). En d'autres termes, les objectifs de l'entreprise artisanale sont, avant tout, ceux de l'artisan qui la crée. Par ailleurs, on sait que les objectifs stratégiques de l'entreprise ne répondent pas forcément ou pas seulement à une logique économique de développement de l'entreprise, notamment par rapport au souci d'indépendance, au désir de réalisation personnelle et au maintien du caractère familial de l'entreprise ${ }^{8}$. Pour toutes ces raisons, il convient de

7. On a souligné que les nombreuses études sur l'entrepreneuriat observent une influence du dirigeant sur le fonctionnement et la stratégie de l'entreprise, bien plus marquée dans le contexte de la PME que dans les grandes organisations (Saporta, 1989; Julien et Marchesnay, 1989).

8. Bauer (1993) propose une analyse qui va dans le même sens en qualifiant le dirigeant de $\mathrm{PME}$ «d'homme à trois têtes», obéissant à une triple rationalité: économique (accroître les résultats de l'entreprise), politique (renforcer son pouvoir) et familiale (aider sa famille et ses enfants). Ce modèle des trois têtes permet alors de rendre compte du comportement des chefs d'entreprises et de comprendre tant leurs décisions quotidiennes que les options stratégiques, engageant durablement l'entreprise, qu'ils privilégient. 
prendre en compte le projet d'entreprendre de l'artisan et les objectifs stratégiques qui lui sont associés pour éclairer l'identité de l'entreprise. Dès lors, c'est principalement à partir de variables éclairant les motivations qui ont poussé l'artisan à s'installer à son compte, sa vision stratégique (au sens de Saporta,1988), ses objectifs stratégiques prioritaires, la manière dont il interprète ses besoins pour développer son entreprise et la manière dont il conçoit la formation que l'on abordera les divergences au regard de ce pôle.

Dans l'artisanat, le projet d'entreprendre semble tellement se fonder essentiellement sur l'exercice d'un métier que la principale caractéristique identitaire de chaque entreprise artisanale peut s'interpréter comme une focalisation sur le métier (au sens de Hoffsteter et Ramanantsoa, 1981). Selon ces auteurs, le concept de focalisation apporte un éclairage révélateur sur l'élément fondamental de l'identité d'une entreprise. Dans leurs travaux, les trois objets possibles de focalisation d'une identité (le leader, l'activité, un comportement) sont présentés comme mutuellement exclusifs. Pour ce qui concerne l'entreprise artisanale, nous souhaitons défendre l'idée que ce n'est pas forcément le cas, en proposant un quatrième objet de focalisation:le métier, comme synthèse des trois autres. Le métier de l'entreprise exerce donc une influence forte sur certains éléments qui contribuent à l'émergence, à la consolidation et au maintien de l'identité de l'entreprise. Cette relation entre métier et identité peut d'abord être envisagée en référence à des éléments tangibles, le métier (entendu au sens de la manière dont l'activité exercée) interagissant, en premier lieu, avec la structure de l'entreprise, et conditionnant, vraisemblablement en grande partie, l'écologie organisationnelle.

Cependant, l'existence d'une identité artisanale commune à toutes les entreprises de ce microcosme fait que leurs caractéristiques structurelles, et notamment la taille, peuvent être considérées comme une «conséquence» ou la résultante de cette identité artisanale ${ }^{9}$. À ce titre, elles ne participent que modérément à la différenciation identitaire d'une entreprise artisanale par rapport à l'autre. Pour cette raison, on préfère aborder cette influence à partir de l'interaction du métier avec les autres éléments du système identitaire de l'entreprise et notamment avec l'imaginaire organisationnel et les productions symboliques. L'imaginaire organisationnel d'une entreprise correspond, en effet, à l'image interne (c'est-à-dire l'image partagée par

9. On fait ici référence à l'influence qu'exerce le secteur des métiers en tant qu'institution, notamment en matière de définition légale des critères d'appartenance à l'artisanat. Compte tenu de ce poids institutionnel, la taille des entreprises (moins de 10 salariés) ainsi que la manière dont le métier doit être exercé (manuellement et avec une participation du chef d'entreprise à la production) sont des caractéristiques communes à toutes les entreprises artisanales et ne peuvent que dans une moindre mesure rendre compte de leur spécificité identitaire. 
tous les membres de l'entreprise) du métier, de la manière «idéale» dont il doit être exercé et dont l'entreprise doit être agencée pour ce faire. Dans l'entreprise artisanale, l'imaginaire organisationnel est celui de l'artisan et peut être approché à partir de la perception et de la représentation de son métier et de son entreprise que se forge l'artisan. De même, pour ce qui est des productions symboliques (les relations interindividuelles et la culture dans l'entreprise artisanale), elles sont la résultante de la manière particulière dont l'activité est exercée (le mode artisanal) et plus précisément de la manière dont l'artisan conçoit et met en œuvre son exercice. Cette dimension du vécu de l'identité peut ainsi également être approchée à partir de la représentation que se fait l'artisan des relations qui doivent exister entre les membres de l'artisanat et des éléments culturels qu'ils doivent partager ${ }^{10}$.

Pour toutes ces raisons, la perception du métier peut être substituée à ces trois sous-systèmes (structure, imaginaire organisationnel et systèmes d'actions). Dans l'artisanat, le métier, c'est avant tout un savoir-faire manuel dont l'apprentissage s'effectue dans le temps, grâce à une pratique quotidienne, qui se transmet et se compose de tâches diversifiées. Le savoir-faire tacite faisant une large place aux «astuces et tours de main», chaque artisan est susceptible de l'exercer d'une façon différente d'un autre. En revanche, les critères perçus qui lui confèrent sa spécificité peuvent être communs à tous les artisans.

En référence aux travaux consacrés à l'analyse des ressources et des connaissances (Winter, 1987;Arrègle, 1995; Reix, 1995), l'opérationnalisation de cette dimension se fera à partir de la manière dont l'artisan perçoit son métier sur un continuum entre tacite et codifiée.

Enfin, notre cadre d'analyse doit également intégrer le cadre de fonctionnement du système de gestion que l'on tente d'approcher, c'est-à-dire son environnement. Compte tenu de son rôle déterminant sur le système de gestion des TPE et PME (Julien et Marchesnay, 1988), l'environnement doit être envisagé comme un des éléments contribuant à l'identité de l'entreprise. Cela d'autant plus que l'identité d'une organisation et sa différenciation par rapport aux autres se construit également par rapport à l'environnement. Pour ce qui est de la manière dont l'influence de ce dernier «entre» dans l'entreprise, là encore, le filtre du dirigeant intervient: conformément à la

10. À cet égard, Thévenet $(1986,1992)$ considère que le métier est l'un des aspects les plus importants de la culture d'une entreprise: «Le métier est sans doute une des sources qui permet le plus étroitement de répondre aux exigences de notre définition de la culture à savoir un patrimoine de compétences développées pour répondre aux problèmes d'adaptation à l'environnement et d'intégration interne de l'organisation» (Thévenet, 1986, p. 88). 
théorie initialement énoncée par Weick (1979), l'environnement et ses évolutions, ses opportunités ou ses menaces n'existent et ne sont perçues par le dirigeant qu'à travers la représentation que celui-ci s'en forge. Ce ne sont donc pas les caractéristiques objectives de l'environnement mais plutôt celles auxquelles le dirigeant prête attention qui forment la spécificité identitaire d'une entreprise ${ }^{11}$. On retiendra ainsi la perception de l'environnement par le dirigeant comme le dernier élément ou pôle constitutif du cadre d'analyse du système identitaire de l'entreprise artisanale. L'opérationnalisation de cette dernière dimension sera faite en distinguant «trois niveaux» d'environnement (micro, méso, macro) et en demandant aux artisans de juger de l'influence des évolutions relatives à chacun de ces niveaux.

\subsection{Les configurations théoriques du système identitaire de l'entreprise artisanale et les hypothèses à tester}

En prenant appui sur les apports des travaux sur l'artisanat mais aussi sur la PME et la TPE, on peut envisager, pour chacun des pôles ci-dessus, différents «états ou formes théoriques ». Si l'on raisonne, pour la clarté de l'exposé, de manière dichotomique en opposant des «types contraires» ou plutôt des extrêmes de continuum, les choses peuvent être résumées comme suit:

- en ce qui concerne le dirigeant de l'entreprise artisanale, deux profils types peuvent s'opposer: d'un côté, l'individu issu du milieu artisanal, qui adhère aux valeurs et au mode de comportement qui est la norme dans le secteur des métiers et qui se considère comme un technicien; de l'autre, l'entrepreneur dont l'appartenance au secteur des métiers est quasiment fortuite (son activité relève de cette institution), étranger à sa culture et qui se considère comme un manager et un gestionnaire.

- de même, en matière de projet d'entreprendre et d'objectifs stratégiques, on peut opposer l'entrepreneur-artisan (au sens de Smith, 1967) ou PIC (selon la distinction de Julien et Marchesnay, 1988), motivé par l'exercice d'un métier, qui privilégiera la reproduction et l'entrepreneur-opportuniste (Smith) ou CAP (Marchesnay), motivé par la puissance, le pouvoir, l'argent, qui cherchera à développer et faire évoluer son entreprise.

11. On fait ici référence au processus d'«enactment» décrit par Weick (1979), dans lequel l'environnement n'existe, pour le dirigeant, que parce qu'il y prête attention. Dès lors, en sélectionnant inconsciemment les caractéristiques de l'environnement qu'il va identifier et donc en procédant à un «enactment», le dirigeant crée luimême ses propres contraintes et celles de son entreprise. 
- pour ce qui est de la perception ou de la représentation du métier, on opposera une conception du savoir-faire privilégiant sa dimension tacite, voire artistique et sa non-reproductibilité, à celui qui, au contraire, pense que son savoir-faire est majoritairement codifiable et/ou codifé, industrialisable.

- enfin, dans le domaine de la perception de l'environnement, en face d'une certaine «myopie» (look in), et d'une vision neutre de l'environnement (sans influence particulière de ce dernier), on trouvera une certaine attention ou vigilance aux opportunités et menaces de l'environnement (look out), une conscience de leur existence et une proactivité.

Il est alors possible de distinguer au moins deux types de système identitaire, que l'on appellera configurations identitaires théoriques, diamétralement opposés. Ces configurations sont présentées dans le tableau 2 à partir de leurs caractéristiques contingentes.

Ces différentes configurations théoriques permettent maintenant de faire émerger les hypothèses qui découlent de notre cadre général d'analyse.

La première hypothèse que l'on souhaite vérifier est celle de l'existence et de la prédominance d'une configuration particulière de ce système identitaire, au sein du secteur des métiers. C'est la question de cette existence que l'hypothèse suivante explicite en se déclinant en quatre sous-hypothèses.

Hypothèse 1. Au sein du secteur des métiers, la configuration de type I (l'artisan) existe et est majoritaire par rapport aux autres configurations possibles. En d'autres termes, il existe une homogénéité de chacun des pôles du modèle, d'une entreprise artisanale à l'autre. Cela se traduit par le fait que les artisans et leurs entreprises partagent des caractéristiques communes et donc qu'il existe et prédomine:

H 1.1 un «profil artisanal» du dirigeant;

H 1.2 un «projet d'entreprendre artisanal et des objectifs stratégiques artisanaux»;

H 1.3 une «perception artisanale de l'environnement»;

H 1.4 une «perception artisanale du métier».

La deuxième hypothèse renvoie à la place centrale qu'occupe le dirigeant au sein du système identitaire de son organisation et au fait qu'il préside aux relations entre les différents pôles de ce système. Ce rôle de «filtre» permet d'envisager qu'en fonction du profil du dirigeant (artisanal ou non), les caractéristiques de chacun des pôles du modèle sont plus ou moins proches 


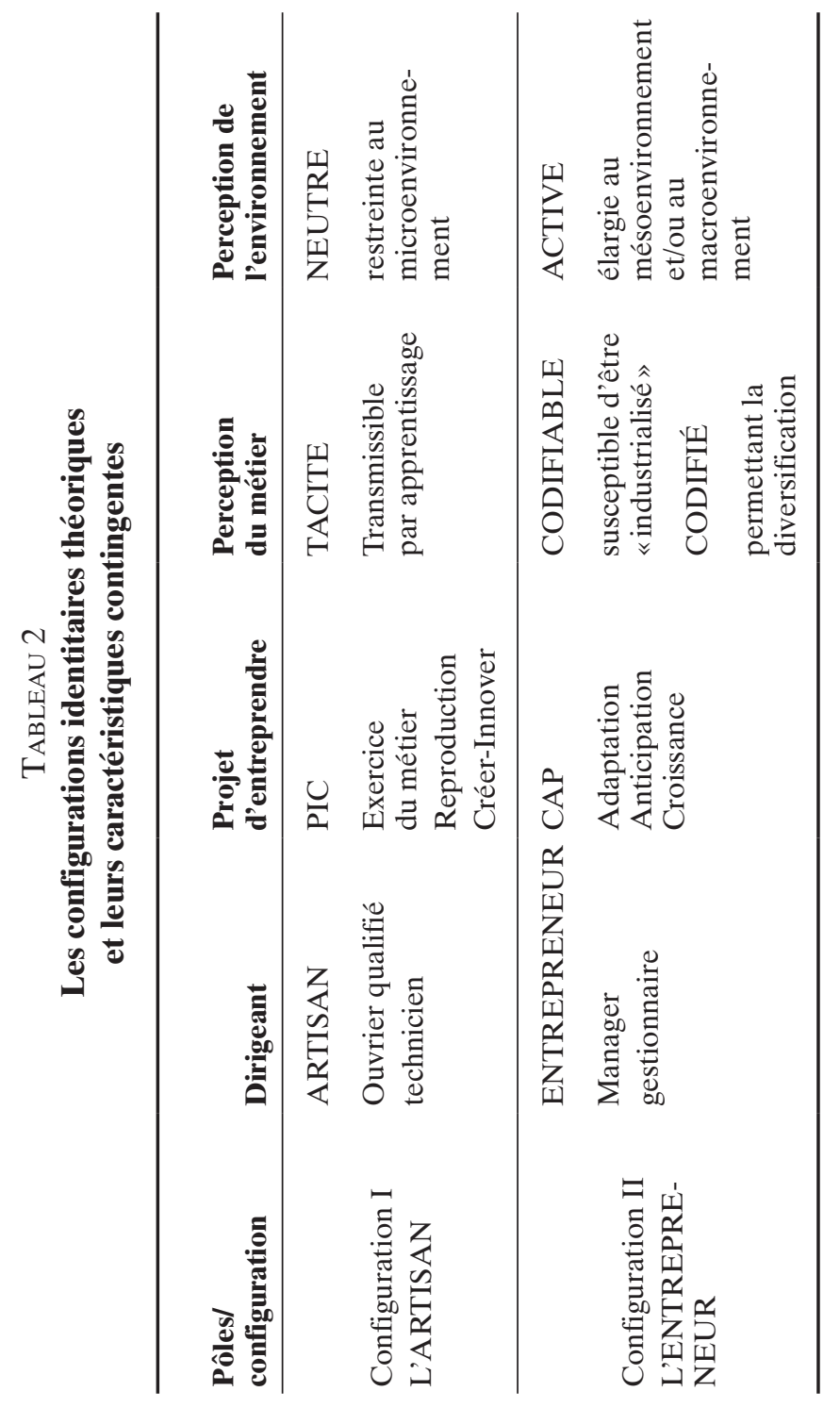

Revue internationale P.M.E., vol. 19, $\mathrm{n}^{\text {os }}$ 3-4, 2006 
de «l'idéal-type artisanal». En d'autres termes, il s'agit ici de vérifier l'importance et l'influence de l'artisan chef d'entreprise dans la constitution de l'identité de son entreprise. Cela nous amène à formuler cette hypothèse.

Hypothèse 2. À un profil du dirigeant de l'entreprise conforme à celui de l'idéal-type artisanal sont associés des pôles du système identitaire possédant des caractéristiques similaires à celles majoritairement observées dans l'artisanat. À l'inverse, plus le profil du dirigeant s'écartera du profil artisanal majoritaire et plus les caractéristiques des autres pôles différeront de celles correspondant à l'idéal-type.

La spécificité d'un système identitaire réside également dans l'interaction particulière entre les sous-systèmes qui le constituent. Sur les plans conceptuel et méthodologique, c'est même l'interaction simultanée entre les quatre pôles de notre modèle qui permet d'en avoir une vision dynamique. Il convient donc de se pencher sur ces interactions. En ce qui concerne la première interaction, la littérature sur les PME et les TPE nous enseigne que les buts, objectifs et finalités de l'artisan sont majoritairement orientés vers la pérennité et donc le maintien, voire la survie de l'entreprise. Parallèlement, l'entreprise artisanale entretient des relations d'un type particulier (caractérisée par une certaine myopie, voire une clôture) avec son environnement. À l'inverse, dans les organisations orientées vers la croissance, le développement est associé à une certaine vigilance à l'égard des mutations environnementales. Qu'en est-il réellement dans l'artisanat et comment cela se traduit-il sur le système identitaire? Ce double constat et ce questionnement sont à l'origine de cette troisième hypothèse.

Hypothèse 3. À des objectifs stratégiques et un projet d'entreprendre traduisant une réticence au développement est associée une perception neutre (influence faible ou nulle sur le fonctionnement et l'évolution de l'entreprise) des éléments de l'environnement et de leurs évolutions. À l'inverse, à des objectifs stratégiques et un projet d'entreprendre orientés vers le développement sera associé un environnement perçu comme ayant une influence active sur l'entreprise.

En ce qui concerne la seconde interaction, les mêmes bases théoriques que la précédente permettent d'envisager une dépendance entre deux pôles du système identitaire de la PME et de la TPE: la manière dont le métier est «vécu», c'est-à-dire la manière dont l'artisan souhaite l'exercer et l'exerce réellement, quotidiennement et le projet d'entreprendre à l'origine de la création de l'entreprise ainsi que les objectifs stratégiques qui en découlent. Afin de vérifier ce lien, nous formulons une quatrième hypothèse. 
Hypothèse 4. À des objectifs stratégiques et un projet d'entreprendre traduisant une réticence au développement est associée une perception «artisanale du métier» (conforme à l'idéal-type en la matière). À l'inverse, à des objectifs stratégiques et un projet d'entreprendre orientés vers le développement est associée une perception du métier différente de celle constitutive de l'identité artisanale.

S'il est possible d'identifier et de valider les deux relations précédentes, alors il faut aussi envisager, pour confirmer la représentation systémique de l'entreprise artisanale, une interdépendance entre la perception du métier et la perception de l'environnement. Nous devons donc également vérifier une cinquième hypothèse.

Hypothèse 5. À une perception «artisanale» du métier (tacite ou codifiable) est associée une perception «artisanale» de l'environnement (neutre). À l'inverse, à une perception «non artisanale» du métier (codifié) est associée une perception de l'environnement différente de celle constitutive de l'identité artisanale (active).

\section{2. Étude empirique des caractéristiques identitaires des entreprises artisanales de Haute-Savoie}

\subsection{Méthodologie}

Afin de vérifier la pertinence de notre construction conceptuelle, une étude quantitative a été menée. Dans ce but, un protocole a été élaboré en collaboration avec la Chambre de métiers de la Haute-Savoie qui était partie prenante dans l'étude et fournissait notamment l'accès au terrain. Les principales étapes sont les suivantes:

- le choix des variables et la rédaction d'un questionnaire: afin de garantir la validité interne et la fiabilité de l'outil de recueil de l'information, l'élaboration du questionnaire a été effectuée en collaboration avec les agents du service Animation économique de la Chambre de métiers et le cabinet de consultant MDR (Marketing Développement Recherche) qui gérait le Panel $74^{12}$.

12. Le Panel 74 est un échantillon d'entreprises artisanales représentatif (sur les critères de taille des entreprises, d'activité exercée et d'ancienneté de l'entreprise) de l'artisanat en Haute-Savoie, constitué, géré et animé par un cabinet-conseil pour le compte de la Chambre de métiers de Haute-Savoie. Il a pour vocation de permettre à la Chambre de métiers de suivre l'évolution de l'activité artisanale dans son département et de recueillir des informations sur des thèmes spécifiques. Les artisans qui le constituent s'engagent à répondre aux questionnaires (trois par an) envoyés par leur Chambre consulaire. 
- l'envoi par la poste de ce questionnaire aux artisans du Panel 74. Deux raisons principales justifient le choix de l'enquête par voie postale. La première tient à la possibilité d'atteindre simultanément un nombre conséquent d'artisans répartis sur toute la Haute-Savoie en limitant le coût de la démarche. La seconde tient au fait que l'on disposait, grâce à la collaboration de la Chambre de métiers, de cet «échantillon captif» garantissant un taux de retour satisfaisant.

- la relance téléphonique auprès des artisans n'ayant pas renvoyé le questionnaire dans un délai de 15 jours. Au total, le taux de retours (environ $60 \%$ ) enregistré dans le cadre de cette enquête par voie postale a été très satisfaisant: sur les 600 questionnaires envoyés, plus de 350 ont été retournés et 346 purent être saisis sur informatique.

- la saisie informatique des questionnaires (logiciel Sphinx) et la réalisation des analyses statistiques correspondantes à la vérification des hypothèses (logiciels Sphinx et SPSS).

En ce qui concerne les typologies, pour chacun de ces pôles, la démarche de classification des 346 observations selon les variables relatives à chacun a été standardisée et suit les deux étapes classiques que nous détaillons ci-dessous.

La première étape est la réduction des données par l'analyse factorielle qui vise à structurer les variables, c'est-à-dire à résumer l'information en remplaçant les variables initiales par un nombre plus petit de variables composites ou facteurs (Evrard, Pras et Roux, 1993). Trois temps jalonnent cette étape:

- la vérification des données, qui permet de s'assurer que l'utilisation de l'analyse factorielle est pertinente. Cette vérification a été effectuée, d'une part, avec le test de sphéricité de Bartlett et, d'autre part, avec la mesure de Kaiser-Meyer-Olkin dite «d'adéquation de l'échantillonnage».

- l'extraction des facteurs à partir de l'analyse en composantes principales, qui a pour objectif de retenir le nombre approprié d'axes ou de facteurs structurants l'information. Deux méthodes ont également été utilisées: celle du critère de Kaiser (1974) et le Scree Test de Catell ${ }^{13}$ ou règle de «l'éboulis».

13. La règle d'arrêt du Scree Test de Cattell conduit à éliminer les facteurs situés après un changement de concavité de la courbe des valeurs propres des variables, c'est-à-dire ceux dont la mise à l'écart n'entraîne qu'une perte minimale d'information. 
- l'interprétation des facteurs, après rotation du plan factoriel (selon la méthode la plus courante, la méthode Varimax), qui se fait à partir des contributions des variables aux axes et, plus précisément, à l'aide des variables dont la corrélation avec les axes (le poids factoriel) est la plus élevée.

La seconde étape est l'identification de groupes différenciés et l'interprétation de la typologie. Afin d'identifier la partition optimale, la pertinence respective de plusieurs scénarii a été évaluée à la lumière d'une succession de critères qualitatifs et statistiques. Pour ce qui est des critères qualitatifs utilisés ici, il s'agit de la capacité de la typologie à «reproduire les solutions passées» ou à s'approcher du modèle théorique, de l'homogénéité de l'effectif des groupes, du sens de la typologie et, enfin, du critère de parcimonie conduisant à privilégier la solution proposant le plus petit nombre de groupes. Pour ce qui est des critères statistiques, on s'est appuyé sur l'analyse de la variance entre les groupes sur les axes factoriels selon le nombre de classes (F de Fischer), sur l'analyse de la capacité discriminante des axes en fonction du nombre de groupes (lambda de Wilks pour identifier la typologie qui minimise la variance intragroupe et maximise la variance intergroupes) et, enfin, sur la capacité prédictive de chaque typologie possible.

\subsection{Les caractéristiques des pôles du système identitaire artisanal}

Dans un premier temps, nous avons cherché à documenter successivement, à partir de nos observations empiriques, les quatre pôles du système identitaire de l'entreprise artisanale. La démarche est donc essentiellement centrée sur la validation, ou l'infirmation de la première hypothèse et des sous-hypothèses qui en découlent. Compte tenu de l'ampleur de l'information à restituer, on ne présente ici que les principaux résultats menant à la vérification des hypothèses.

\subsubsection{Le profil de l'artisan}

La vérification de l'existence d'un profil artisanal type s'est faite à partir de neuf variables ( $c f$. tableau de l'annexe). Les résultats obtenus à l'issue de l'analyse factorielle ont permis de supprimer les variables V2 (date de création), V4 (type de création), V 77 (sexe) et V 82 (situation avant l'installation) qui conduisaient à des résultats inconsistants. 
L'extraction des facteurs nous incite à retenir deux axes restituant $62,8 \%$ de la variance. Les profils des artisans dans l'échantillon se différencient donc selon deux grandes dimensions ou facteurs: «Jeunesse ou ancienneté dans l'artisanat» et «Formation professionnelle».

La procédure itérative de constitution de groupes nous amène à retenir une partition en trois classes qui donne lieu à l'interprétation que nous présentons ci-après.

- La classe 1 regroupe des artisans (124) qui sont d'un âge intermédiaire, voire jeunes (relativement à la moyenne de l'échantillon) et qui dirigent leur entreprise depuis une dizaine d'années (à la date de l'enquête). Ces chefs d'entreprise possèdent un niveau d'étude plutôt bas (niveau CAP/BEP) et ne sont qu'exceptionnellement titulaires du brevet de maître artisan. Par ailleurs, leur formation professionnelle est proche de l'idéal-type artisanal dans ce domaine: apprentissage sur le tas et en entreprise. On les appellera «les débutants ».

- La classe 2 regroupe des artisans (91) nettement plus âgés que les précédents et qui dirigent également leur entreprise depuis plus longtemps (plus de 25 ans à la date de l'enquête). Ils possèdent un niveau d'étude similaire ou à peine supérieur à celui du premier groupe. En revanche, ils semblent disposer d'une qualification professionnelle plus riche: ils sont en moyenne plus nombreux à détenir le titre de maître artisan; leur expérience et leur compétence technique ont été acquises de manière plus variée. C'est dans ce groupe que l'on compte le plus de compagnons du Tour de France. On les appellera «les maîtres artisans ».

- La classe 3 est composée d'artisans (84) dont le profil est assez proche de celui du premier groupe sur les critères d'ancienneté dans l'artisanat: âge moyen, durée de direction d'environ dix années. En revanche, ils se distinguent fortement des autres sur les critères de formation: niveau d'étude élevé puisqu'au moins équivalent au bac (c'est certainement dans leurs rangs que l'on trouve les ingénieurs de notre échantillon), formation professionnelle moins proche de l'apprentissage institutionnel. On les appellera «les ingénieurs».

Notre typologie introduit donc une nuance dans la dichotomie envisagée lors de la formulation des hypothèses en faisant émerger de nos observations un profil intermédiaire de chef d'entreprise artisanale: il existe deux groupes d'individus (la classe 1,124 «débutants», la classe 2, 91 «maître artisans ») dont le profil correspond à l'idéal-type théorique artisanal. Ils se 
distinguent d'abord sur les critères d'ancienneté dans l'artisanat et d'âge, mais également sur la formation professionnelle suivie et, de manière assez logique, sur l'expérience et la compétence dans le métier. Pour ce qui est de la classe 3 , elle regroupe les 84 «ingénieurs » dont le profil est le plus éloigné de cet idéal-type. Au regard du nombre d'artisans (215) proches de l'idéaltype, on considérera que l'hypothèse H 1.1 est validée.

\subsubsection{Les perceptions du métier}

L'identification de perceptions différenciées des métiers pratiqués par les artisans de l'échantillon, notamment en termes de caractéristiques, de savoirfaire et de compétences nécessaires pour les exercer, a été effectuée à partir de neuf variables détaillées en annexe. Ces variables avaient également pour vocation de faire le lien entre les caractéristiques perçues du métier et son caractère «codifiable ou non codifiable». Les différences de perception du métier s'organisent autour de trois facteurs : la «Créativité du savoir-faire», la «Variété du savoir-faire» et la «Rareté du savoir-faire».

La procédure itérative de constitution de groupes et les comparaisons des différentes solutions acceptables nous amènent à retenir à nouveau la partition en trois classes et à en proposer l'interprétation suivante.

- La classe 1 regroupe les 131 artisans qui perçoivent le métier qu'ils exercent comme étant essentiellement artistique, faisant une large part à des tâches de conception variées, complexes, plutôt manuelles. Ils considèrent également que la part de service et de l'adaptation à la demande est très importante. En référence au cadre théorique, et sur la base des travaux de Reix (1995), cette classe regroupe les artisans détenant des savoir-faire non encore codifiés mais éventuellement codifiables, tout au moins en partie. Il s'agit vraisemblablement des métiers en mutation (coiffure, boulangerie, etc.) qui évoluent de manière incrémentale ou radicale en fonction du progrès technique, des innovations technologiques et des changements de comportement des consommateurs et utilisateurs. Puisque le savoir-faire est considéré comme relativement courant et transmissible, on désignera cette classe par l'expression «perception évolutive ou codifiable du savoir-faire».

- La classe 2 est celle des 54 artisans qui n'attribuent pas de caractéristiques propres au métier qu'ils exercent. Leur perception se situe dans la moyenne si ce n'est, et cela constitue la seule particularité de ce groupe, qu'ils jugent leur métier peu complexe techniquement, peu manuel et demandant la réalisation de tâches très peu variées. Dans 
ce groupe, les savoir-faire détenus semblent plus courants, codifiés ou en tout cas codifiables. Il s'agit vraisemblablement des métiers (décolletage...) appartenant à des secteurs d'activité dans lesquels les entreprises artisanales sont en concurrence avec des firmes plus importantes et dans lesquels elles se spécialisent sur des créneaux particuliers tout en mobilisant des savoir-faire répandus et maîtrisés. Puisque les caractéristiques perçues du savoir-faire correspondent à celles d'un métier industrialisé, d'exécution (peu complexe, peu manuel, dont les tâches ont très peu variées et dans lequel la part de service est faible), on désignera cette classe par l'expression "perception sans particularité ou codifiée du savoir-faire».

- La classe 3 est composée de 82 artisans dont la perception du métier met en avant la rareté de leur savoir-faire, la difficulté à le transmettre ainsi qu'une grande importance du service et de l'adaptation à la demande. La principale différence avec ceux du premier groupe est qu'ils considèrent leur métier comme peu artistique et comportant une faible part de conception. Ces artisans semblent détenteurs de savoir-faire que l'on pourrait qualifier de «purement artisanaux», faisant une large part au «tour de main». On peut imaginer qu'il s'agit d'activités traditionnelles, éventuellement en déclin, stables techniquement, principalement tournées vers le consommateur final et satisfaisant une demande de produits uniques, individualisés et / ou très différenciés (ébénisterie...). Puisque les principales caractéristiques perçues du savoir-faire sont la rareté et la difficulté à le transmettre, on désignera cette classe par l'expression «perception artisanale ou tacite du savoir-faire».

En résumé, on a donc deux groupes (1 et 3) proches de la perception ou de la représentation artisanale du métier exercé et du savoir-faire maîtrisé (tacite et/ou codifiable). Paradoxalement, ils se distinguent principalement sur la rareté du savoir-faire, sa dimension artistique ainsi que sur la fréquence des tâches de conception. Si on les associe, on observe bien une prédominance de cette perception artisanale. Malgré cette nuance, on considérera alors l'hypothèse H 1.2 comme validée.

\subsubsection{Les perceptions de l'influence de l'environnement}

La recherche de perceptions différenciées de l'influence de l'environnement s'est faite à partir de 15 variables ( $c f$. l'annexe), répondant à un découpage de l'environnement en trois niveaux. 
L'extraction des facteurs «autour» desquels se structurent les données nous amène à considérer que l'influence de l'environnement sur leur entreprise et sur leur métier est perçue par les artisans à travers quatre principales dimensions (facteurs): l' «Influence des évolutions techniques », $l^{\prime}$ «Influence institutionnelle», l'«Influence des conseillers» et l'«Influence du marché et des partenaires».

La typologie retenue comporte quatre groupes. Son interprétation conduit à proposer une classification empirique qui enrichit la dichotomie théorique en introduisant, entre des extrêmes, deux groupes aux profils intermédiaires. On a alors un continuum en quatre groupes aux effectifs relativement proches:

- Le groupe des «myopes» (68 artisans) qui semblent imperméables aux évolutions environnementales et semblent se rapprocher du profil type de l'artisan tel que notre cadre théorique l'envisageait;

- Le groupe des «institutionnels» (49 artisans) qui ont conscience du fait que l'environnement n'est pas neutre pour leur entreprise mais ont une vision très institutionnelle de son influence;

- Le groupe des «techniciens» (67 artisans), un peu plus ouverts que les précédents sur leur environnement mais surtout beaucoup plus attentifs aux mutations qui concernent leur métier et sa codification (progrès technique et innovations);

- Le groupe des «vigilants» (61 artisans) qui sont les plus ouverts sur l'influence de leur environnement, dans toutes ses composantes et qui l'analysent vraisemblablement comme étant à la fois porteur d'opportunités et de menaces.

Cette classification diffère donc également de celle attendue logiquement et qui opposait deux profils d'artisans : ceux que l'on pourrait qualifier de «myopes» et ceux que l'on pourrait qualifier de «vigilants». À nouveau, l'hypothèse d'une prédominance d'un profil artisanal type correspondant aux «myopes» doit donc être nuancée mais pas réfutée. L'hypothèse $\mathrm{H} 1.3$ est considérée comme validée.

\subsubsection{Les projets d'entreprendre et les objectifs stratégiques}

Pour ce pôle, la démarche a été réalisée à l'aide de trois séries de variables (motivations à la création d'une entreprise artisanale, objectifs prioritaires concernant le devenir de l'entreprise et actions de développement). En 
raison de la nature différente des variables relatives à ces trois dimensions ${ }^{14}$, on a procédé en deux temps: la construction d'une typologie portant sur les actions de développement envisagées et considérées comme adaptées à leur métier par chacun des artisans de l'échantillon puis la mise en relation de cette typologie avec les deux autres dimensions définies pour ce pôle du modèle. La description des classes ci-après intègre toutes les informations obtenues à l'issue de ces traitements.

Pour la première étape, 19 variables relatives aux actions de développement susceptibles d'être jugées pertinentes par nos artisans ont été retenues ( $c f$. l'annexe). Il se dégage trois axes autour desquels s'organise la perception des actions stratégiques adaptées à la situation et aux objectifs de nos artisans : «Expansion et croissance», «Gestion des coûts », «Positionnement produits».

Pour la typologie, une partition en quatre groupes différenciés d'artisans est retenue. On a alors à nouveau un continuum dans lequel se positionnent deux profils intermédiaires entre deux extrêmes correspondant pour l'un à «l'artisan PIC ou entrepreneur artisan» et, pour l'autre, à «l'artisan CAP ou opportuniste».

- Le groupe des «entrepreneurs expansionnistes» (134 artisans), qui considèrent que la croissance est la voie stratégique la plus adaptée et sont donc les artisans que l'on jugera les plus offensifs, stratégiquement parlant. Ils semblent alors s'opposer radicalement au profil théorique de l'artisan tel que notre cadre théorique l'envisageait, d'autant plus que leur objectif prioritaire est de développer leur activité. Cependant, ils ont créé leur entreprise dans le but d'exercer le métier qu'ils aiment et pour être autonomes. En cela, ils ne sont donc pas aussi différents de l'idéal-type artisanal.

- Le groupe des «artisans centrés sur leurs produits» (51 artisans), qui expriment également une certaine offensivité. Cependant, la démarche stratégique qu'ils privilégient est différente ou plus «limitée» que celle du groupe précédent. Elle est avant tout orientée vers la spécialisation, l'exploitation d'un savoir-faire et l'innovation produit. Ces artisans jugent donc qu'une stratégie de «niche» ou de différenciation (Porter, 1986) est la plus adaptée à leur situation

14. Dans notre étude, les objectifs stratégiques et le projet d'entreprendre des artisans ont été «mesurés » à partir de trois questions : deux sont des variables qualitatives (motivation à la création de l'entreprise et objectifs stratégiques prioritaires), la troisième est en fait une série d'échelles de Likert correspondant à des variables quantitatives. Ces deux types de variables ne pouvant être traités statistiquement de manière similaire, cela justifie la démarche en deux temps.

Revue internationale P.M.E., vol. 19, nos 3-4, 2006 
et à leur métier. Ils se sont installés d'abord pour créer leur propre emploi, mais également à des fins d'autonomie et d'indépendance. Paradoxalement, ils projettent prioritairement de développer leur activité.

- Le groupe des «artisans rationalistes» (88 artisans), qui pourraient être qualifiés «d'artisans gestionnaires» puisque les orientations stratégiques qu'ils privilégient relèvent de la diminution des coûts de production et de l'amélioration de la gestion interne de leur entreprise. Si l'on se réfère aux stratégies génériques de Porter, cela peut s'interpréter comme une sensibilité relevant de la domination globale par les coûts, à la différence près que, dans le cas des entreprises artisanales, il s'agit vraisemblablement d'une attitude plus défensive qu'offensive. Ces artisans ont simultanément des objectifs de maintien et de développement. Leur motivation d'installation est majoritairement reliée au désir de s'accomplir personnellement dans l'exercice d'un métier qu'ils aiment.

- Le groupe des «artisans statiques» (74 artisans), qui n'expriment pas de sensibilité stratégique et ne privilégient aucune action. Ce sont les artisans qui apparaissent comme les plus proches du profil théorique ou de l'idéal-type artisanal: pour eux, la détention d'un savoir-faire suffit pour maintenir l'entreprise et cette conception engendre un comportement stratégique passif et statique. Ces derniers ont plutôt des objectifs de maintien de l'activité jusqu'à la retraite, voire, pour certains, de cessation de l'activité à court terme. Créer leur emploi et être indépendant sont les motivations qui les animaient au moment de l'installation à leur compte.

En définitive, en intégrant les autres séries de variables, les profils qui apparaissaient comme différenciés ne le sont pas autant que la typologie sur les actions de développement privilégiées le montrait. En matière de projet d'entreprendre et d'objectifs stratégiques, les individus qui composent notre échantillon sont donc majoritairement des artisans. En conséquence, on considérera que l'hypothèse H 1.4 est validée.

Au terme de cette description de chacun des pôles du système identitaire artisanal, on dispose des résultats suivants:

- La confirmation de l'hypothèse H 1.1 par la mise en évidence de trois profils d'artisans (débutants, maîtres artisans, ingénieurs) dont deux sont proches de l'idéal-type artisanal. 
- La confirmation de l'hypothèse H 1.2 par la caractérisation de trois perceptions différentes du métier (évolutive, sans particularités, artisanale) dont deux sont également assez proches de celle conjecturée.

- La confirmation de l'hypothèse H 1.3 même si les résultats sont plus mêlés. En effet, la typologie met en évidence quatre perceptions différenciées de l'influence de l'environnement et donc quatre attitudes par rapport à ce dernier (myopes, institutionnels, techniciens, vigilants). Schématiquement, parmi ces quatres, les trois premières sont conformes ou, en tout cas, proches de l'idéal-type artisanal puisqu'elles correspondent à une fermeture ou à une ouverture limitée au micro- ou mésoenvironnement. La quatrième est celle qui se caractérise le plus par une ouverture sur la plupart des dimensions de l'environnement.

- La confirmation de l'hypothèse H 1.4 par la mise en évidence de quatre types d'orientations stratégiques qui se répartissent sur un continuum allant des artisans statiques aux artisans expansionnistes. Si l'on tient compte des informations supplémentaires que nous apportent les variables concernant les motivations à la création et l'objectif prioritaire, les artisans privilégiant le statu quo (statiques et rationalistes) sont majoritaires par rapport aux artisans stratégiquement plus offensifs (centrés produits et expansionnistes).

Compte tenu de ces éléments et malgré les nuances relevées par rapport à la dichotomie envisagée sur chaque pôle, on considérera que l'hypothèse $\mathrm{H} 1$ est validée.

\subsection{Les configurations types du système identitaire artisanal}

L'objectif principal de cette dernière étape est d'examiner les hypothèses restantes $(\mathrm{H} 2, \mathrm{H} 3, \mathrm{H} 4, \mathrm{H} 5)$ à la lumière des résultats obtenus dans le point précédent pour, au final, se prononcer sur la réalité d'une coexistence des deux configurations identitaires théoriques. On procédera donc en deux temps: premièrement, en cherchant à établir des liens entre les différents groupes des typologies construites sur les quatre pôles de notre modèle; deuxièmement, en dressant un portrait des configurations identitaires qui se dessinent empiriquement. Sur le plan de la méthode, les résultats présentés ici ont été obtenus en croisant les typologies entre elles et en évaluant leur 
dépendance par un test du chi carré15. Dans un souci de «parcimonie» de l'information, seuls les résultats synthétiques (sans les tableaux statistiques) des analyses sont indiqués.

\subsubsection{L'influence du profil du dirigeant et l'interdépendance entre les pôles du système identitaire}

En ce qui concerne tout d'abord l'influence prépondérante du dirigeant sur les autres pôles du système identitaire $(\mathrm{H} 2)$, les tris croisés entre profil du dirigeant et perception du métier, perception de l'environnement et objectifs stratégiques/projet d'entreprendre conduisent à rejeter l'hypothèse H 2. Les caractéristiques du dirigeant (l'appartenance plus ou moins marquée au milieu artisanal, l'âge, le fait d'avoir ou non suivi une formation par l'apprentissage, le fait de diriger l'entreprise depuis plus ou moins longtemps ou encore le fait d'être titulaire d'un diplôme de maître artisan) n'influent pas ou peu sur les autres éléments du noyau identitaire de l'entreprise artisanale. Deux explications peuvent être avancées: soit notre typologie différencie statistiquement des profils au sein d'une dimension dans laquelle c'est plutôt l'homogénéité qui domine; soit le poids de l'identité institutionnelle est si fort que, quel que soit son profil initial, le dirigeant se «coule dans le moule».

En ce qui concerne, ensuite, l'interdépendance conjecturée des orientations stratégiques avec les autres pôles identitaires, les résultats des tableaux croisés autorisent les commentaires suivants:

- l'hypothèse selon laquelle, dans l'artisanat, plus on est myope, moins on est expansionniste, et inversement, est globalement vérifiée. L'hypothèse $\mathrm{H} 3$ est donc validée.

- la perception la plus artisanale du métier est associée au comportement stratégique le plus artisanal et de manière symétrique, la perception la moins artisanale du métier (évolutive) correspond également à un comportement stratégique différent de l'idéal-type artisanal (sans particularité). De ce point de vue, on considérera donc que l'hypothèse $\mathrm{H} 4$ est validée.

- dans l'artisanat, plus on a une perception évolutive de son savoirfaire et du métier que l'on exerce et plus on est sensible ou plus on

15. Afin d'optimiser l'utilisation du test du chi carré, chaque groupe de chacune des typologies a d'abord été transformé en une variable fermée du type oui/non (deux typologies à trois groupes plus deux typologies à quatre groupes, soit 14 variables dichotomiques). Dans un second temps, selon les relations prévues par les hypothèses, ces variables ont été croisées deux à deux, afin de mesurer précisément la dépendance entre elles.

Revue internationale P.M.E., vol. 19, nºs 3-4, 2006 
ressent l'influence des modifications de l'environnement dans lequel on évolue. L'inverse n'étant pas statistiquement vérifié, l'hypothèse H 5 n'est donc que partiellement validée. Ce résultat peut également s'interpréter comme le fait que l'attention aux éventuelles modifications de l'environnement est d'autant plus grande que l'on pense que notre métier est susceptible d'évoluer. Ce dernier résultat tend aussi à confirmer l'effet de système et donc à valider l'approche de l'entreprise artisanale en termes de système identitaire particulier.

Enfin et en plus des hypothèses présentées, on a souhaité «mesurer» l'influence de l'activité exercée (selon les postes de la nomenclature officielle des activités artisanales) sur les pôles du système identitaire de l'entreprise artisanale. Les résultats des tris croisés amènent aux constats suivants: si l'on a bien une dépendance entre certains pôles du système identitaire (profil du dirigeant, perceptions du métier et de l'environnement) et l'activité exercée, cette dépendance entre le système identitaire et l'activité n'est pas totale puisque les différentes orientations stratégiques identifiées au sein de l'artisanat sont «équitablement (ou quasiment) réparties». En outre, si l'on regroupe les 18 activités en quatre postes (bâtiment, production, alimentation, services), selon la nomenclature artisanale des métiers, on constate que ces relations ne sont plus, systématiquement, statistiquement vérifiées. En d'autres termes, il n'y a pas d'homogénéité identitaire au sein des activités d'une même catégorie. Dans le bâtiment, par exemple, les maçons ont une perception sans particularité, voire évolutive de leur métier et savoir-faire alors que les artisans de la couverture-plomberie-chauffage en ont une perception très artisanale.

C'est donc dans les particularités de chaque profession qu'il faut chercher tel ou tel positionnement identitaire. Plus précisément, en matière de perception du métier, mais aussi de perception de l'environnement, il semble que ce soit la stabilité ou, au contraire l'instabilité technique et technologique (innovations, nouveaux procédés, nouveaux matériaux et matières, etc.) qui soit un facteur d'homogénéité ou d'hétérogénéité. Les menuisiers-serruriers ont ainsi une perception évolutive de leur métier tout en étant attentifs aux évolutions techniques réelles dans leurs professions (menuiserie préfabriquée, aluminium, PVC, serrures de sécurité, etc.). 


\subsubsection{Les configurations empiriques du système identitaire artisanal}

Dans cette dernière étape, il s'agit maintenant de se prononcer sur la coexistence des deux configurations identitaires théoriques. L'ensemble des résultats empiriques précédents autorise l'identification, au sein de notre échantillon, de ces deux profils types. En effet, à partir des relations statistiquement vérifiées entre les typologies et les groupes qui les composent, on observe bien deux configurations particulières du système identitaire de l'entreprise artisanale. Ces configurations se différencient sur trois des quatre pôles de ce système: la perception du métier, la perception de l'environnement, les objectifs stratégiques et le projet d'entreprendre. L'hypothèse $\mathrm{H} 2$ concernant l'influence du profil du dirigeant sur le système n'étant pas validée, ces deux configurations ne se différencient pas sur ce pôle profil du dirigeant.

La figure 3 propose une visualisation graphique de la position de ces deux configurations types sur les trois dimensions concernées. On distingue ainsi:

- «l'artisan» dont les «coordonnées» sont entre «myopes» $(\mathrm{M})$ et «institutionnels» (I) sur l'axe de la perception de l'environnement, entre «centrés sur le produit» $(\mathrm{CP})$ et «statiques» $(\mathrm{S})$ sur l'axe des objectifs stratégiques et projet d'entreprendre et, enfin, entre «évolutive» (E) et «artisanale» (A) sur l'axe de la perception du métier;

- «l'entrepreneur» dont les « coordonnées» sont entre «techniciens» (T) et «vigilants» (V) sur l'axe de la perception de l'environnement, entre «expansionnistes» $(\mathrm{E})$ et «rationalistes» $(\mathrm{R})$ sur l'axe des objectifs stratégiques et projet d'entreprendre et, enfin, «sans particularité » (SP) sur l'axe de la perception du métier.

Afin de quantifier le nombre d'artisans ou d'entreprises de notre échantillon qui correspondent à ces critères, une variable «profil» a été construite à l'aide du logiciel Sphin $x^{16}$. Elle permet de constater que, dans notre échantillon, on compte 40 individus correspondant strictement au profil «artisan » et 16 individus correspondant strictement au profil «entrepreneur». Ces chiffres relativement faibles s'expliquent de trois manières: tout d'abord, il s'agit de types purs, c'est-à-dire de systèmes identitaires «cumulant» des caractéristiques bien précises et interdépendantes. Ensuite, 290 questionnaires ont été

16. Une variable profil est une variable fermée créée par la combinaison de plusieurs variables et permettant de repérer et de regrouper les réponses apportées à plusieurs questions. Chacune de ses modalités répond à un ensemble de conditions et définit alors un profil. Notre variable compte deux modalités: artisan et entrepreneur, regroupant les individus classés simultanément selon leur position sur les trois dimensions du profil. 
exclus de cette dernière analyse parce qu'ils contenaient au moins une nonréponse à l'une des questions se rapportant aux trois typologies concernées ici (perception du métier, perception de l'environnement, objectifs stratégiques). Enfin, on soulignera que si nos observations avaient été équitablement réparties dans la matrice correspondante à cette analyse ( 8 colonnes et 3 lignes, soit 24 cases), c'est-à-dire s'il n'y avait pas eu de configuration identitaire dominante, on aurait eu, en moyenne, 2,3 observations par case.

Figure 3

\section{Les profils types de l'artisanat}

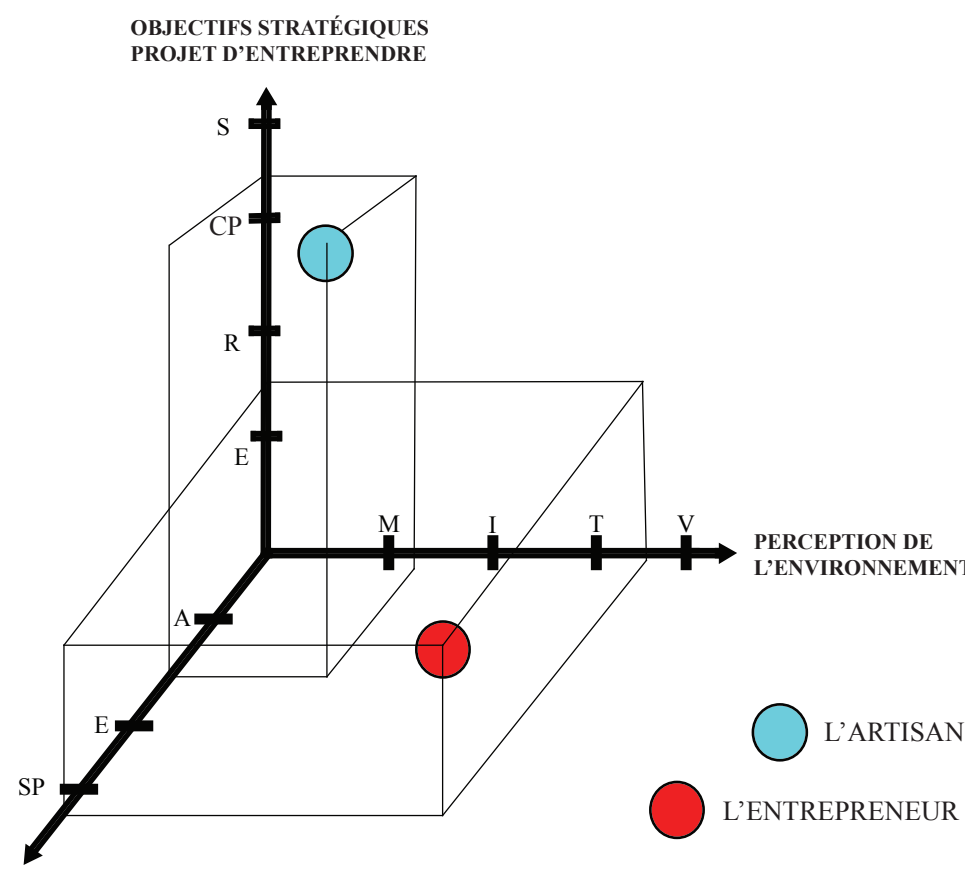

PERCEPTION DU MÉTIER

En résumé, sur les artisans classés dans les quatre typologies (environ 200 puisque ceux qui n'ont pas répondu à l'une des questions ont été exclus de l'analyse), seuls 56 correspondent à des «types purs» sur le plan identitaire. 


\section{Conclusion}

L'ensemble de ce travail s'est inscrit dans une perspective qui vise à améliorer la connaissance de l'entreprise artisanale et de son identité. Partant du constat que cette forme d'organisation et son comportement stratégique sont relativement mal connus du gestionnaire, la mobilisation de la métaphore identitaire et son adaptation au cas particulier de la firme artisanale a débouché sur une représentation conceptuelle novatrice du système identitaire de l'entreprise artisanale. Les analyses menées à partir d'une enquête auprès de 346 artisans ont ensuite montré qu'il était possible, au sein de cet échantillon, de « découvrir des régularités » (au sens de Koenig, 1993), de les ordonner et de faire apparaître des configurations types de ce système identitaire. L'ensemble de ces résultats nous conduit alors à valider empiriquement cette représentation. Il est donc possible de mobiliser le concept d'identité et plus généralement le paradigme identitaire, initialement développés pour les grandes entreprises et de l'adapter aux TPE. On peut donc considérer que l'on dispose maintenant d'une grille de lecture «identitaire » qui contribue à une meilleure intelligibilité de l'entreprise artisanale, en tant que forme d'organisation spécifique, de son fonctionnement et de son pilotage.

Bien entendu, il convient de souligner les principales limites inhérentes, d'une part, aux choix conceptuels et, d'autre part, aux partis pris méthodologiques. En ce qui concerne les choix conceptuels, notons que l'adaptation de l'approche identitaire au cas de l'entreprise artisanale est probablement à l'origine de raccourcis et de simplification. Ensuite, le double, voire triple rattachement thématique aboutit aussi certainement à un balancement entre deux, voire trois types de littérature, celle sur l'artisanat, celle sur la PME et celle sur l'identité. Sur le plan méthodologique, il faut tout d'abord rappeler que, dans ce type d'enquête, la validité de «la mesure » est liée à la pertinence des questions posées. En outre, il faut ajouter que l'on a principalement travaillé sur la perception des artisans, difficile à «contrôler» par voie postale. Enfin, la question de la représentativité de l'échantillon des panélistes pourrait être posée dans la mesure où, au-delà des questions de représentativité statistique, on peut se demander si des artisans volontaires pour faire partie d'un panel ne sont pas déjà sensiblement différents des autres.

Au regard de l'intérêt pour les instances représentatives de l'artisanat, il convient de réaffirmer le caractère exploratoire d'une telle démarche. En conséquence, la possibilité d'opérationnaliser des résultats obtenus reste limitée. Néanmoins, ces instances et plus particulièrement les Chambres de métiers peuvent trouver dans ce travail matière à réflexion. Dans un contexte de concurrence exacerbée, où l'artisanat n'est plus un secteur refuge,l'objectif de survie des entreprises artisanales nécessite que l'on se pose la question de 
l'adéquation entre l'identité artisanale et les conditions d'exercice des activités (environnement). Bien qu'aucune mesure de la performance ou de la compétitivité ne soit intégrée dans notre étude, le constat pourrait ressembler à une déconnexion relative entre les valeurs communes aux artisans et celles véhiculées par le discours sur l'entreprise et sa réussite.

Revue internationale P.M.E., vol. 19, $\mathrm{n}^{\text {os }}$ 3-4, 2006 


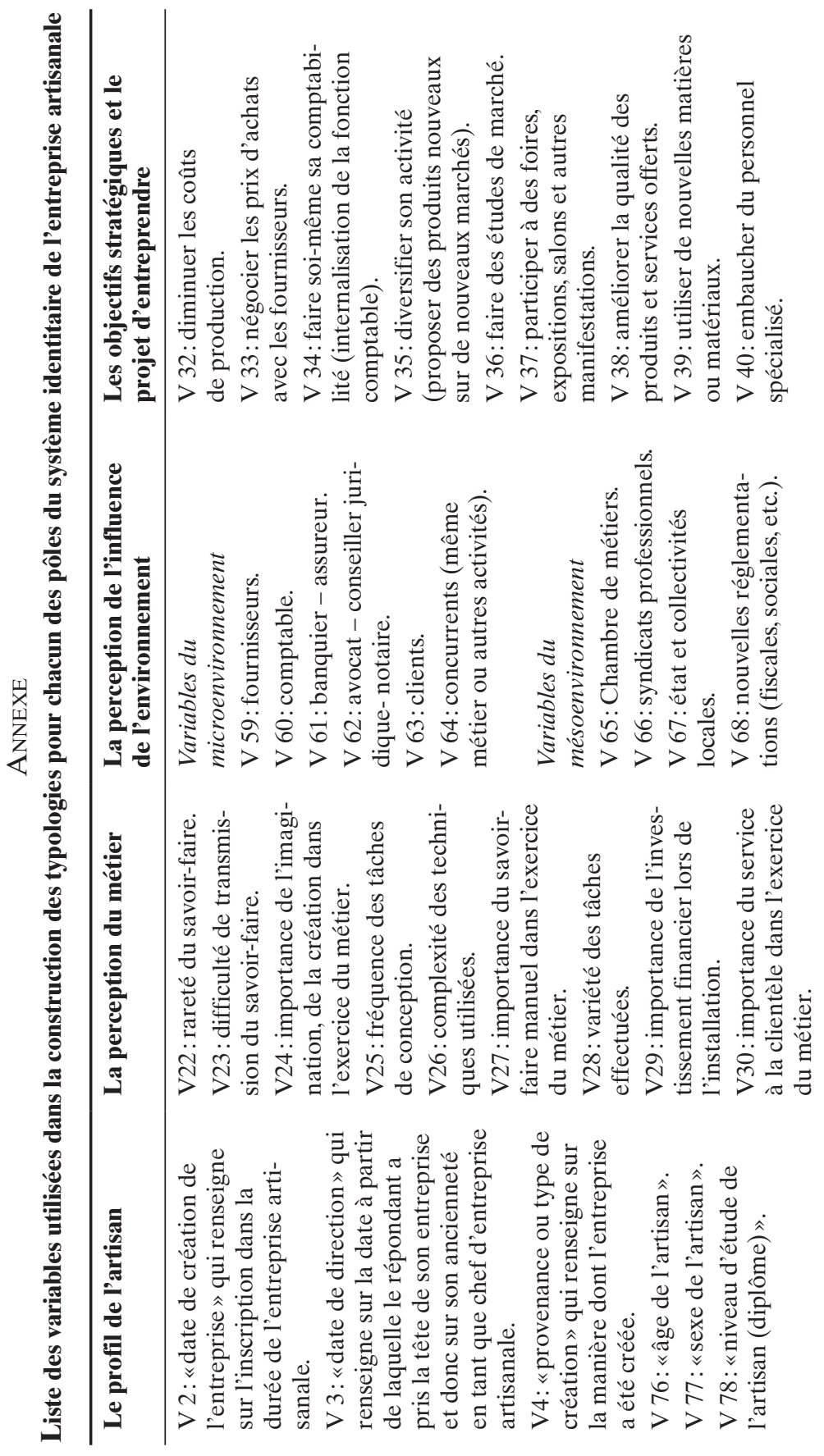

Revue internationale P.M.E., vol. 19, $\mathrm{n}^{\text {os }}$ 3-4, 2006 


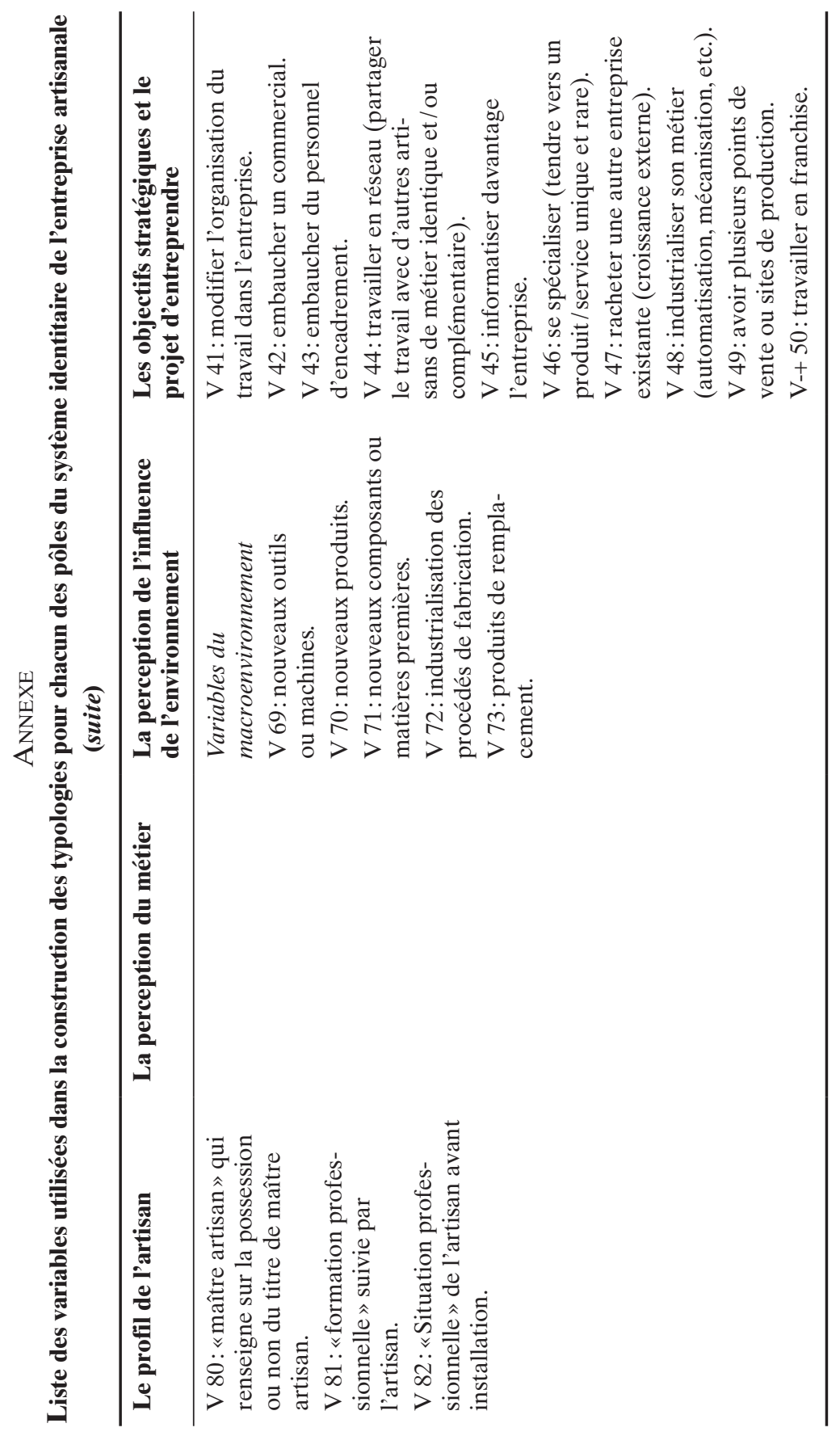

Revue internationale P.M.E., vol. 19, $\mathrm{n}^{\text {os }}$ 3-4, 2006 


\section{Bibliographie}

Abric, J.C. et R. Mardella (1983), «Les représentations sociales de l'artisan et de l'artisanat», GIFRESH, Paris, Centre d'études et de perfectionnement de l'artisanat et des métiers.

Arena, R., J.P. Arrighi, P. Cuturello et P.M. Romani (1982), « Rapport de recherche sur l'artisanat de production et de sous-traitance industrielle en Lot-etGaronne», LATAPSES, CNRS, Université de Nice, juillet.

ArRègle, J.L. (1995), «Le savoir et l'approche resource based: une ressource et une compétence », Revue française de gestion, septembre-octobre, ${ }^{\circ}$ 105, p. 84-94.

Auvolat, M. (1990), «Études et recherches sur l'artisanat: analyse de la production des années 80 », IRPEACS-CNRS, Ministère du Commerce et de l'Artisanat, Direction de l'artisanat.

Auvolat, M., M. Lavigne et A. Mayère (1985), L'artisanat en France, réflexion générale et prospective, Paris, La Documentation française.

BAUER, M. (1993), Les patrons de PME entre le pouvoir, l'entreprise et la famille, Paris, InterÉditions, $245 \mathrm{p}$.

BÉNARD, C.F. (1927), La notion d'artisan dans la loi et dans les faits, Paris, Droit.

Chaudieu, G. (1982), Artisans et commerçants, Paris, Éditions CIEM, 158 p.

Debré, M. (1934), L'artisanat classe sociale, Paris, Droit.

Direction des EnTREPRises Commerciales, ARTisanales ET DE SERVICES - DEcas? (2004), Les chiffres clefs de l'artisanat en France.

Evrard, Y., B. Pras et E. Roux (1993), Market: Études et recherches en marketing. Fondements et méthodes, Paris, Nathan.

Fourcade, C. (1993), «Stratégie de réseau: un modèle stratégique de l'internationalisation des petites entreprises», Actes du Colloque PME-PMI: Développement international, p. 303-323, organisé par le CER et le GREPME, Aix-en-Provence.

Froger, J. et D. Weiller (1986), «À la recherche du nouvel artisan», Cahiers du Centre scientifique et technique du bâtiment, mai.

Hellriegel, D., J.W. Slocum et R.W.Woodman (1989), Management des organisations, Bruxelles, De Boeck Université.

Hoffsteter, C. et B. Ramanantsoa (1981), «La maîtrise de l'identité par la gestion du processus de focalisation: une nouvelle donnée stratégique? », Direction et Gestion, no 4, p. 19-33.

JAEger, C. (1982), Artisanat et capitalisme, l'envers de la roue de l'histoire, Paris, Payot.

Jarniou, P. et C. LÉGer (1989), «L'évolution des métiers de l'artisanat du bâtiment, ou comment gérer tradition et modernité», Centre d'études et de perfectionnement de l'artisanat et des métiers, Paris, juillet.

Julien, P.-A. (dir.) (1994), Les PME, bilan et perspectives, Paris, Economica.

Revue internationale P.M.E., vol. 19, nºs 3-4, 2006

(C) 2006 - Presses de l'Université du Québec

Édifice Le Delta I, 2875, boul. Laurier, bureau 450, Québec, Québec G1V 2M2 • Tél.: (418) 657-4399 - www.puq.ca

Tiré de: Revue internationale P.M.E., vol. 19, nos $3-4$, sous la direction de Louis Raymond - PME1903N

Tous droits de reproduction, de traduction et d'adaptation réservés 
Julien, P.-A. et M. Marchesnay (1988), La petite entreprise, principes d'économie et de gestion, Paris, Vuibert, coll. «Gestion».

KAISER, H.F. (1974), «An index of factorial simplicity», Psychometrica, $\mathrm{n}^{\circ} 39$.

KAPFERER, J.N. (1988), «Maîtriser l'image de l'entreprise : le prisme d'identité», Revue française de gestion, novembre-décembre, p. 76-82.

Koenig, G. (1993), «Production de la connaissance et constitution des pratiques organisationnelles», Revue de gestion des ressources humaines, $\mathrm{n}^{\circ}$ 9, p. 4-17.

Larçon, J.P. et R. ReItTer (1979a), «L'identité de l'entreprise, un facteur-clé de sa survie », Direction et Gestion, n ${ }^{\circ}$ 3, p. 11-16.

LARÇON, J.P. et R. ReItTER (1979b), Structures de pouvoir et identité de l'entreprise, Paris, Nathan.

Laufer, R. et B. Ramanantsoa (1982), "Crise d'identité ou crise de légitimité ?», Revue française de gestion, septembre-octobre, p. 18-26.

LETOwsKi, A. (1987), «Les systèmes socio-culturels de l'artisanat face aux mutations », Université Paris VI Dauphine.

LOUART, P. (1985), «Les artisans face à la gestion de leur personnel. Pratiques sociales et logiques sous-jacentes», Commissariat général du Plan.

Marchesnay, M. (1980), «Sur la petite entreprise», Revue d'économie industrielle, $\mathrm{n}^{\circ} 11$.

MARChesnay, M. (1982), «Is small so beautiful?», Revue d'économie industrielle, $\mathrm{n}^{\circ} 19$.

Marchesnay, M. (1985), «L'artisanat: un monde en changement», Cahiers Enseignement et Gestion.

Marchesnay, M. (1991), «De la théorisation en sciences de gestion», Économie et Sociétés, Série Sciences de gestion, nº 17, avril, p. 195-209.

MARChESNAY, M. (1993), «PME, stratégie et recherche», Revue française de gestion, $\mathrm{n}^{\circ}$ 95, septembre-octobre, p. 70-76.

Marchesnay, M. (2003), «La petite entreprise : sortir de l'ignorance», Revue française de gestion, Dossier spécial TPE, ${ }^{\circ}$ 144, juin, p. 107-118.

Marchesnay, M. (2005), «Éloges complexes de la diversité», Atelier AIREPME, Montpellier, mai.

Mignot, G. (1980), Les perspectives de développement de l'artisanat et de la petite entreprise, Paris, La Documentation française.

Mucchielli, A. (1992), L'identité, Paris, Presses universitaires de France.

NizARD, G. (1983), «Identité et culture de l'entreprise », Harvard-L'Expansion, hiver, p. $90-106$.

PORTER, M. (1986), L'avantage concurrentiel, Paris, InterÉditions.

ReIX, R. (1995), «Savoir tacite et savoir formalisé dans l'entreprise », Revue française de gestion, $\mathrm{n}^{\circ} 105$, septembre-octobre, p. 17-28.

Revue internationale P.M.E., vol. 19, n ${ }^{\text {s }} 3-4,2006$ 
Rojot, J. et A. Bergmann (1989), Comportement et organisation, Paris, Vuibert, coll. «Gestion».

SAPORTA, B. (1988), Stratégies pour la PME, Paris, Montchrestien, coll. «Entreprendre».

SAPORTA, B. (1989), «Stratégie des petites et moyennes entreprises», Encyclopédie de gestion, Paris, Economica, p. 2729-2754.

Sмith, N.R. (1967), «The entrepreneur and his firm: the relationship between type of man and type of company», Bureau of Business and Economic Research, Michigan State University.

Thévenet, M. (1986), Audit de la culture d'entreprise, Paris, Les Éditions d'Organisation.

Thévenet, M. (1992), «Culture d'entreprise», dans Encyclopédie du Management, tome I, Paris, Vuibert.

Voisin, J.R. (1986), «L'entreprise de bâtiment change: quel sera son nouveau visage ?», Le Moniteur, 17 janvier.

WeIck, K.E. (1979), The Social Psychology of Organizing, New York, McGraw-Hill.

WinTER, S.G. (1987), «Knowledge and competence as strategy assets», dans D. Teece (dir.), The Competitive Challenge, Cambridge, Ballinger.

ZARCA, B. (1982), «La rationalité économique des artisans», Consommation revue de socio-économie, $\mathrm{n}^{\circ}$ 1, p. 3-38.

ZARCA, B. (1986), L'artisanat français, du métier traditionnel au groupe social, Paris, Economica.

ZARCA, B. (1987), Les artisans: gens de métier, gens de parole, Paris, L'Harmattan, coll. «Logiques sociales».

ZARCA, B. (1988), «Identité de métier et identité artisanale», Revue française de sociologie, $\mathrm{n}^{\circ} 29$, p. 247-273.

Zemor, P. (1984), «Les quatre vérités de la politique d'entreprise», HarvardL'Expansion, printemps, p. 67-72.

Revue internationale P.M.E., vol. 19, $\mathrm{n}^{\text {os }} 3-4,2006$

(C) 2006 - Presses de l'Université du Québec

Édifice Le Delta I, 2875, boul. Laurier, bureau 450, Québec, Québec G1V 2M2 • Tél.: (418) 657-4399 - www.puq.ca 